\title{
3-month daily rifampicin and isoniazid compared to 6- or 9-month isoniazid for treating latent tuberculosis infection in children and adolescents less than 15 years of age: an updated systematic review
}

\section{To the Editor:}

One-third to one-quarter of the world's population is estimated to have latent tuberculosis infection (LTBI) [1]. These infected persons are at risk for developing active disease with a lifetime risk of reactivation estimated to be $5-10 \%$ [2]. Prevention of reactivation of LTBI in population groups with higher risk of progression to active disease than the general population, is critical to end the global TB epidemic [3].

The World Health Organization (WHO) recommends 6-month isoniazid $(6 \mathrm{H})$ monotherapy for the treatment of LTBI in children [4]. However, the effectiveness of $6 \mathrm{H}$ is compromised by low rates of treatment completion and adverse events [5]. A systematic review of the literature on the options for treating LTBI in children was conducted in 2012. The review found out that 3- or 4-month rifampicin (R) and isoniazid $(\mathrm{H})$ combination therapy was as effective as $6 \mathrm{H}$ or $9 \mathrm{H}$ monotherapy with higher completion rate and no evidence of increased hepatotoxicity [6].

Currently, a water-dissolvable and child-friendly fixed-dose combination of $\mathrm{R}$ and $\mathrm{H}$ is available [7]. We thus performed an updated systematic review to evaluate the effectiveness of a $3 \mathrm{RH}$ combination therapy compared with a $6 \mathrm{H}$ or $9 \mathrm{H}$ monotherapy for the treatment of LTBI in children and adolescents $<15$ years of age. The outcome measurements were: TB incidence, mortality, adverse events, treatment adherence and treatment completion and drug resistance. The literature search was conducted in PUBMED and EMBASE databases, from December 2012 to January 2017, to identify studies published after the previous review [6]. Abstract books of international conferences, and reference lists of included studies were also searched.

Literature search strategies were developed using text words: 1) "latent tuberculosis"; 2) "isoniazid"; 3) "rifampicin"; 4) "treatment" or "prophylaxis"; 5) "children", and related terms. Studies were selected based on participants (children and adolescents $<15$ years of age); interventions (a $3 \mathrm{RH}$ daily combination therapy); comparators (a $6 \mathrm{H}$ or $9 \mathrm{H}$ daily monotherapy). The review was limited to English, French and Spanish languages.

We assessed the quality of individual studies in terms of their study design and execution. Relative risks (RRs), comparing relative effects of $3 \mathrm{RH}$ with $6 \mathrm{H}$ or $9 \mathrm{H}$, were calculated using Stata 14: Data Analysis and Statistical Software (StataCorp LP, College Station, TX, USA). We could not conduct a meta-analysis due to the limited number of included studies and their clinical and methodological heterogeneity. Findings were reported in accordance with the PRISMA (preferred reporting items for systematic reviews and meta-analyses) statement [8].

@ERSpublications

Treatment of latent tuberculosis infection in children with a 3-month daily rifampicin and isoniazid regimen is safe with better compliance, adherence and completion rate than a 6- or 9-month isoniazid monotherapy http://ow.ly/x28Z30jDfCE

Cite this article as: Assefa Y, Assefa Y, Woldeyohannes S, et al. 3-month daily rifampicin and isoniazid compared to 6- or 9-month isoniazid for treating latent tuberculosis infection in children and adolescents less than 15 years of age: an updated systematic review. Eur Respir J 2018; 52: 1800395 [https://doi.org/ $10.1183 / 13993003.00395-2018]$. 


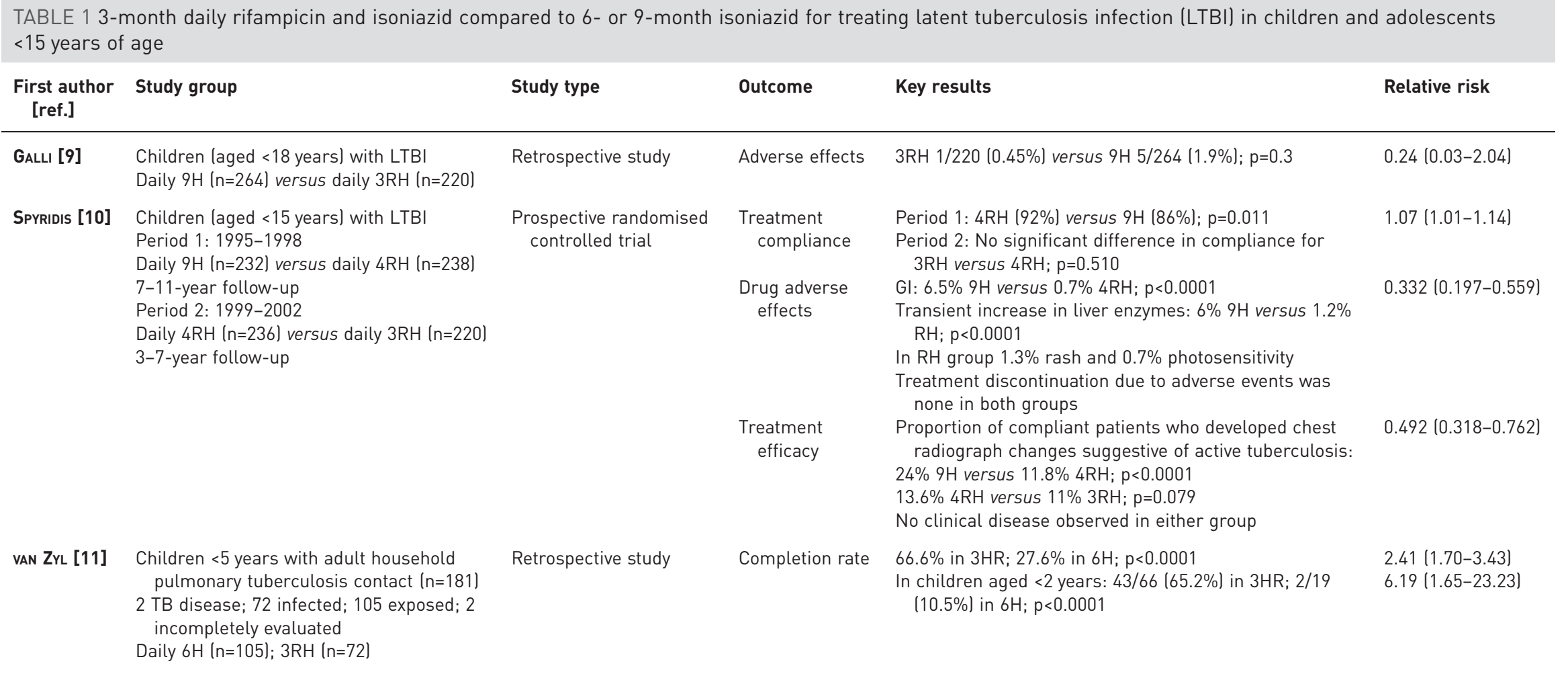


The search identified 281 unique hits. We excluded 17 studies due to duplication and 240 studies by title and abstract review. Full text review was conducted for 24 studies, and we found only one study that can be included in the current updated systematic review [9]. We identified two papers from the previous review $[10,11]$; the other papers were not relevant for the current review due to either their population or interventions (not 3RH). Thus, three papers were finally included in the current review.

GALLi et al. [9] "reported the results of a multicentre retrospective review, using records of children (aged $<18$ years) diagnosed with active and latent TB between January 2010 and December 2012, in 27 health facilities in Italy." SPYRIDIs et al. [10] "published the results of a prospective, randomised, controlled study conducted in 1995-2005 among children aged <15 years of age at Department of Paediatrics in Athens University, Greece. Patients were enrolled during two time periods (period 1, from 1 January 1995 through 31 December 1998; and period 2, from 1 January 1999 through 31 December 2002)." VAN ZYL et al. [11] "reported the results of a retrospective study comparing $3 \mathrm{RH}$ with $6 \mathrm{H}$ in children $<5$ years of age, identified as household contacts of adult pulmonary TB, conducted from January 1996 to September 2003, in Cape Town, South Africa (table 1)." A judgement on the quality of these studies indicated that there was a high risk of bias in each study.

Treatment efficacy: the proportion of compliant patients who developed chest radiograph (CXR) changes suggestive of active TB was $24 \%$ in $9 \mathrm{H}$ and $11.8 \%$ in $4 \mathrm{RH}(\mathrm{p}<0.0001)$ in period 1 , and $13.6 \%$ in $4 \mathrm{RH}$ and $11 \%$ in $3 \mathrm{RH}(\mathrm{p}=0.079)$ in period 2. The risk of development of CXR changes was significantly lower in those given $4 \mathrm{RH}$ compared to $9 \mathrm{H}$ with $\mathrm{RR}$ equivalent to 0.492 (95\% CI 0.318 to 0.762 ). However, there was no clinical disease observed in either group. Data on treatment efficacy were not available from the two observational studies [10].

Adverse events: the rate of gastro-intestinal related adverse events was $6.5 \%$ in $9 \mathrm{H}$ and $0.7 \%$ in $4 \mathrm{RH}$ $(\mathrm{p}<0.0001)$. There was a transient increase in liver enzymes in $6 \%$ of study participants in $9 \mathrm{H}$ compared with $1.2 \%$ in $4 \mathrm{RH}(\mathrm{p}<0.0001)$ with a $\mathrm{RR}$ of adverse events equivalent to 0.332 (95\% CI: 0.197 to 0.559$)$; however, there was no treatment discontinuation due to adverse events in both groups [10]. The rate of liver function impairment was $0.45 \%$ in $3 \mathrm{RH}$ and $1.9 \%$ in $9 \mathrm{H}$ though there was no significant difference $(\mathrm{p}=0.3)[9]$.

Treatment compliance and adherence: treatment compliance was $92 \%$ in $4 \mathrm{RH}$ and $86 \%$ in $9 \mathrm{H}(\mathrm{p}=0.011)$ during period 1 . There was, however, no significant difference in compliance in $3 \mathrm{RH}$ and $4 \mathrm{RH}(\mathrm{p}=0.510)$ during period 2 with a RR $(95 \% \mathrm{CI})$ equivalent to 1.07 (1.01 to 1.14) [10]. Treatment completion rate of $3 \mathrm{RH}(66.7 \%)$ was significantly higher than that of $6 \mathrm{H}(27.6 \%)$ with a $\mathrm{RR}$ equivalent to 2.41 (95\% CI 1.70 to 3.43$)$ [11].

Drug resistance: none of the included studies reported about development of drug resistance following preventive treatment among children and adolescents $<15$ years of age.

In summary, the updated systematic review found that adherence was significantly better in $3 \mathrm{RH}$ than in $6 \mathrm{H}$ or $9 \mathrm{H}$. Children who received $9 \mathrm{H}$ monotherapy were less compliant than those who received short-course combination therapy. No serious drug-related adverse effects were detected; moreover, the risk of side effects was lower in children treated with a $3 \mathrm{RH}$ combination therapy compared to a $9 \mathrm{H}$ monotherapy. Data on preventive efficacy were limited and reported from only one study. Although the study reported no patient who developed clinical disease during the follow-up period, new radiographic findings suggestive of possible active $\mathrm{TB}$ disease were less common in patients who received a $3 \mathrm{RH}$ combination regimens than those treated with a $9 \mathrm{H}$ monotherapy.

There are also other studies, not included in this review due to absence of a control group, which suggested findings consistent with our review that treatment with short-course RH produced a greatly reduced proportion of paediatric notifications of tuberculosis. They were also tolerated without any toxicity [12]. Another cohort study published in 2010 reported that $3 \mathrm{RH}$ has very high efficacy and no significant hepatitis [13].

Even though studies included in this review did not report on the development of drug resistance following TB preventive treatment in children, systematic reviews done mostly among adults showed no evidence of a significant association between development of drug resistance and use of isoniazid or rifamycins for preventive treatment. However, exclusion and diagnosis of active TB among children is difficult. Therefore, it is important to establish a national surveillance system to monitor drug resistance among children receiving preventive treatment [14].

This review has a number of limitations. First, very few studies compared $3 \mathrm{RH}$ with $6 \mathrm{H}$ or $9 \mathrm{H}$ for the treatment of LTBI in children and adolescents $<15$ years of age. Secondly, children were classified as having TB disease based on clinical presentation and/or CXR changes that may result in false positive or 
false negative diagnoses. However, data from a systematic review among adults and children suggest that $3 \mathrm{RH}$ has similar efficacy to $6 \mathrm{H}$ or $9 \mathrm{H}$; therefore, it is plausible that $3 \mathrm{RH}$ has at least similar or better efficacy compared to $6 / 9 \mathrm{H}$ in children as well [15]. Lastly, two of the three studies are from low-TB burden countries and the findings from the studies may not be generalisable to high-TB burden countries.

In conclusion, treatment of LTBI in children with a daily $3 \mathrm{RH}$ regimen is safe with better compliance, adherence and completion rate than a $6 \mathrm{H}$ or $9 \mathrm{H}$ monotherapy. Hence, $3 \mathrm{RH}$ can be considered as a preferable option, compared to isoniazid monotherapy, for treating LTBI particularly in light of availability of child friendly formulations. Nevertheless, more and better-quality evidence is needed on the effectiveness and safety of this regimen.

Yibeltal Assefa $^{1}$, Yalemzewod Assefa ${ }^{1}$, Solomon Woldeyohannes ${ }^{1}$, Yohhei Hamada ${ }^{2}$ and Haileyesus Getahun ${ }^{2}$

${ }^{1}$ School of Public Health, the University of Queensland, Brisbane, Australia. ${ }^{2}$ Global TB Programme, World Health Organization, Geneva, Switzerland.

Correspondence: Yibeltal Assefa. E-mail: y.alemu@uq.edu.au

Received: Feb 232018 | Accepted after revision: April 132018

This is one of a selection of articles published open access, as part of an initiative agreed between the European Respiratory Society and the World Health Organization.

Conflict of interest: None declared.

\section{References}

1 Houben RM, Dodd PJ. The global burden of latent tuberculosis infection: a re-estimation using mathematical modelling. PLoS Med 2016; 13: e1002152.

2 Getahun H, Matteelli A, Abubakar I, et al. Management of latent Mycobacterium tuberculosis infection: WHO guidelines for low tuberculosis burden countries. Eur Respir J 2015; 46: 1563-1576.

3 World Health Organization. The End TB Strategy. Global strategy and targets for tuberculosis prevention, care and control after 2015. Geneva, WHO, 2014.

4 World Health Organization. Recommendations for investigating contacts of persons with infectious tuberculosis in low-and middle-income countries. Geneva, WHO, 2012.

5 Alsdurf H, Hill PC, Matteelli A, et al. The cascade of care in diagnosis and treatment of latent tuberculosis infection: a systematic review and meta-analysis. Lancet Infect Dis 2016; 16: 1269-1278.

6 Gwee A, Coghlan B, Curtis N. What are the options for treating latent TB infection in children? Arch Dis Child 2013; 98: 468-474.

7 World Health Organization. New fixed-dose combinations for the treatment of TB in children. Geneva, WHO, 2016.

8 Moher D, Liberati A, Tetzlaff J, et al. Preferred reporting items for systematic reviews and meta-analyses: the PRISMA statement. PLoS Med 2009; 6: e1000097.

9 Galli L, Lancella L, Tersigni C, et al. Pediatric tuberculosis in Italian children: epidemiological and clinical data from the Italian register of pediatric tuberculosis. Int J Mol Sci 2016; 17: 960.

10 Spyridis NP, Spyridis PG, Gelesme A, et al. The effectiveness of a 9-month regimen of isoniazid alone versus 3 -and 4-month regimens of isoniazid plus rifampin for treatment of latent tuberculosis infection in children: results of an 11-year randomized study. Clin Infect Dis 2007; 45: 715-722.

11 Van Zyl S, Marais B, Hesseling A, et al. Adherence to anti-tuberculosis chemoprophylaxis and treatment in children. Int J Tubercul Lung Dis 2006; 10: 13-18.

12 Ormerod L. Rifampicin and isoniazid prophylactic chemotherapy for tuberculosis. Arch Dis Child 1998; 78: $169-171$.

13 Bright-Thomas R, Nandwani S, Smith J, et al. Effectiveness of 3 months of rifampicin and isoniazid chemoprophylaxis for the treatment of latent tuberculosis infection in children. Arch Dis Child 2010; 95: 600-602.

14 Den Boon S, Matteelli A, Getahun H. Rifampicin resistance after treatment for latent tuberculous infection: a systematic review and meta-analysis. Int J Tuberc Lung Dis 2016; 20: 1065-1071.

15 Zenner D, Beer N, Harris RJ, et al. Treatment of latent tuberculosis infection: an updated network meta-analysis. Ann Intern Med 2017; 167: 248-255. 